\title{
Nonlinear lightwave circuits in chalcogenide glasses fabricated by ultrafast laser
}

\author{
Mingshan Li, ${ }^{1}$ Sheng Huang, ${ }^{1}$ Qingqing Wang, ${ }^{1}$ Hrvoje Petek, ${ }^{2}$ and Kevin P. Chen ${ }^{1, *}$ \\ ${ }^{1}$ Department of Electrical Engineering, University of Pittsburgh, Pittsburgh, Pennsylvania 15261, USA \\ ${ }^{2}$ Department of Physics and Astronomy, University of Pittsburgh, Pittsburgh, Pennsylvania 15260, USA \\ *Corresponding author: kchen@engr.pitt.edu
}

Received October 16, 2013; revised December 9, 2013; accepted December 16, 2013;

posted December 18, 2013 (Doc. ID 199609); published January 30, 2014

\begin{abstract}
This Letter reports a nonlinear directional waveguide coupler written by ultrafast laser in gallium lanthanum sulfide chalcogenide glass. The nonlinear waveguide device is tested with laser pulses input in two orthogonal polarizations, and all optical switching at $1040 \mathrm{~nm}$ between the two coupled waveguides is observed at a peak fluence of $16 \mathrm{GW} / \mathrm{cm}^{2}$. The spectra and autocorrelation measurement from the waveguide outputs show dominant nonlinear effects and negligible dispersion for light propagation in both channels. ( 2014 Optical Society of America

OCIS codes: (350.3390) Laser materials processing; (160.4330) Nonlinear optical materials; (190.4390) Nonlinear optics, integrated optics; (130.4815) Optical switching devices.

http://dx.doi.org/10.1364/OL.39.000693
\end{abstract}

Chalcogenide glasses (ChGs) are a class of important optical materials useful for mid-infrared optical technology and nonlinear optics [1]. A number of micro-fabrication techniques have been applied to produce ChG-based nonlinear optic waveguide devices that exploit their wide transparency window, high nonlinearity, and low multi-photon absorption [2]. A popular fabrication technique for ChG-based waveguide devices is through thinfilm deposition and various lithography schemes [3]. Although a number of excellent waveguide devices have been demonstrated using thin-film based approach, performance of photonic devices is extremely sensitive to properties of ChG films. In many situations, thin optical films produced by state-of-the-art deposition techniques do not possess optical qualities as good as those found in bulk substrates. This challenge in fabrication diminishes the possibility of building three-dimensional photonic structures through the conventional layer-by-layer lithography technique.

Ultrafast laser writing technique is another alternative to produce waveguide devices in $\mathrm{ChG}$ glasses. The unique trait for this technique is its ability to fabricate arbitrary complex structures and three-dimensional (3D) integrated circuits in bulk optical glass with proven optical quality [4]. It has been proven in fabricating both linear $[\underline{5}, \underline{6}]$ and nonlinear $[\underline{7}, \underline{8}]$ optical devices in silica.

Although it is beneficial to use highly nonlinear materials to fabricate nonlinear optical devices, it is also a challenge to process such materials with ultrafast laser irradiation. This is because the focused writing beam with high peak intensity could experience large distortion in the material, resulting in an enlarged modification area and undesired material changes along the direction of the writing beam. However, these challenges could be overcome by minimizing the spatial and temporal distortion of the writing beam by means of beam shaping and temporal pulse tuning. Recently, we have demonstrated ultrafast laser fabrication of high-quality waveguide in ChG glasses with highly symmetric waveguide profile and $0.65 \mathrm{~dB} / \mathrm{cm}$ propagation loss at $1550 \mathrm{~nm}$ [9]. This opens the possibility of producing 3D nonlinear lightwave circuits in bulk materials.
In this Letter, we present a nonlinear directional coupler (NLDC) fabricated in gallium lanthanum sulfide (GLS) chalcogenide glass by ultrafast laser. The selection of GLS glass is based on its good thermal stability and its nontoxic nature $[10,11]$. The performance of the NLDC is investigated at two orthogonal input polarizations. The output power fractions of the two ports of the NLDC vary with the peak intensity of the input laser pulses, and a clear all-optical switching is observed at $16 \mathrm{GW} / \mathrm{cm}^{2}$. The output spectra and the autocorrelation traces from both ports show that the nonlinear effect is dominant in the pulse propagation while the linear dispersion can be neglected.

The NLDC in GLS ChG glass is written using a Coherent RegA 9000 laser system, with chirped laser pulses $(1.7 \mathrm{ps})$ at $800 \mathrm{~nm}$ at a repetition rate of $250 \mathrm{kHz}$. An astigmatic beam shaping technique is used to spatially modify the input beam so as to write symmetric waveguides with low propagation loss in this highly nonlinear material [9]. During the writing, the GLS sample is mounted on a three-axis motion stage (Aerotech) and translated at $1.5 \mathrm{~mm} / \mathrm{s}$ in the direction orthogonal to the writing beam. With its polarization parallel to the writing direction, the writing beam has $328 \mathrm{~nJ}$ pulse energy and is focused by an $80 \times$ objective $(\mathrm{NA}=0.75)$ at $237 \mu \mathrm{m}$ below the sample surface.

The configuration of the ChG coupler and its end facet is observed by optical microscopy. The schematic of the NLDC is shown in Fig. 1 , with microscope images of the merging region shown in inset (a) and the end facet shown in inset (b). The input and output facets of the sample are $10 \mathrm{~mm}$ apart. At both facets, the input/output ports of the two coupler arms are separated by $58 \mu \mathrm{m}$; in transition to the coupling region, double S-bend structures with $32 \mathrm{~mm}$ radius are used; the two arms are evanescently coupled at the center of the sample, where the center-to-center separation is set to $8 \mu \mathrm{m}$ to allow maximum interaction between the two waveguides while minimizing the overlap of the modifications due to ultrafast laser writing; and the coupling length is set to $6 \mathrm{~mm}$. From the microscope image of the end facets, the modified areas of both waveguides are measured $\sim 7.5 \mu \mathrm{m}$ in 


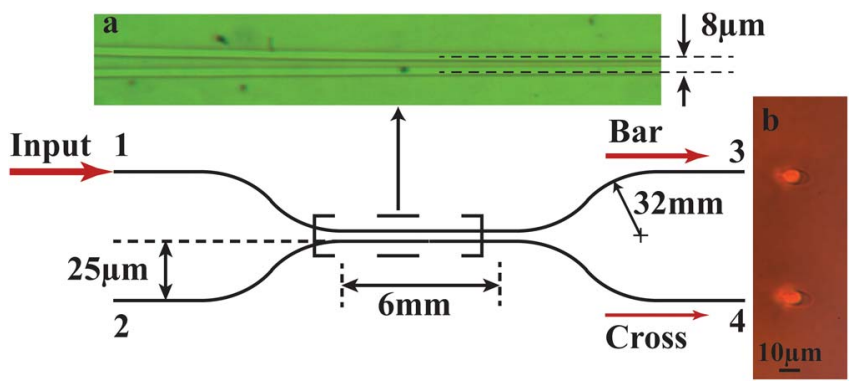

Fig. 1. Schematic of the NLDC and microscope images of the merging region [inset (a)], and the output facet [inset (b)]. In the measurements, the straight through channel is denoted as Bar, and the cross-coupled channel is denoted as Cross.

the direction along the writing beam and $\sim 5.5 \mu \mathrm{m}$ in the orthogonal direction.

The performance of the NLDC is measured using the setup in Fig. 2. Before the measurements, the ChG NLDC is thermally annealed at $235^{\circ} \mathrm{C}$ for an hour to prevent photoinduced effects of the material upon the input of high intensity pulses [2]. The IMPULSE femtosecond laser system is used in the test of the ChG NLDC. It produces laser pulses centered at $1040 \mathrm{~nm}$. The repetition rate of laser pulses is $500 \mathrm{kHz}$ and its pulse width is measured to be 446 fs by an autocorrelator after going through two half-wave plates and a polarizer to control its power and polarization. An aspheric lens with $f=13.86 \mathrm{~mm}, \mathrm{NA}=0.18$ is used to launch laser pulses into the coupler via one of the input ports. The outputs from both ports of the coupler are collected and collimated using another aspheric lens with $f=4.51 \mathrm{~mm}$, $\mathrm{NA}=0.55$. One of the coupler outputs is selected to go through an iris for subsequent measurements. The selected output is split by two beam splitters and sent to a power meter, an optical spectrum analyzer (ANDO OSA AQ6315B), and imaged by a lens with $f=300 \mathrm{~mm}$ on a CMOS camera (Thorlabs DCC1240M). The selected output is also reflected by a mirror on a flip mount to an autocorrelator for pulse width measurement.

As a reference, the ChG waveguides with the same writing parameters are first tested for both input polarizations. With $1.5 \mathrm{~dB}$ reflection loss from the input

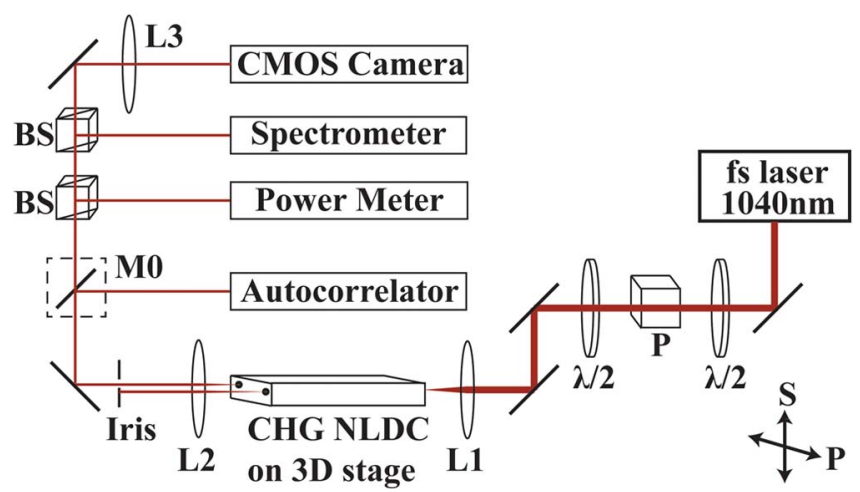

Fig. 2. Experimental setup, BS: beam splitter; L1: aspheric lens, $f=13.86 \mathrm{~mm}$; L2: aspheric lens, $f=4.51 \mathrm{~mm}$; L3: imaging lens, $f=300 \mathrm{~mm}$; M0: mirror on flip mount; $\mathrm{P}$ : polarizer. $\mathrm{P}$ polarization is along the direction of the two couplers outputs; S-polarization is orthogonal to the direction of the two coupler outputs. and output facets of the sample, the insertion losses for the two polarizations are similar, with $4.6 \mathrm{~dB}$ for $p$ polarized input and $4.4 \mathrm{~dB}$ for $s$-polarized input. The near field mode profiles are shown in Fig. 3, and their full width at half-maximum (FWHM) in two orthogonal directions are found by Gaussian fitting. It shows that both $p$-polarized and $s$-polarized pulses propagate in the waveguide at the fundamental mode; the $p$-polarized light shows a better circular symmetry. Besides, both input polarizations experience a large amount of spectral broadening when the input peak intensity increases from 5 to $90 \mathrm{GW} / \mathrm{cm}^{2}$, with the spectral width increasing from about $20 \mathrm{~nm}$ to over $140 \mathrm{~nm}$. The pulse stretching is insignificant for the short propagation length inside the waveguide, but it is related to the input intensity due to the nonlinear index change in the waveguide: for $p$-polarized input, the pulse width is increased to $633 \mathrm{fs}$ at $5 \mathrm{GW} / \mathrm{cm}^{2}$, and to $1004 \mathrm{fs}$ at $90 \mathrm{GW} / \mathrm{cm}^{2}$; for $s$-polarized input, the pulse width is increased to $558 \mathrm{fs}$ at $5 \mathrm{GW} / \mathrm{cm}^{2}$, and to $930 \mathrm{fs}$ at $90 \mathrm{GW} / \mathrm{cm}^{2}$. These results imply that nonlinear effect is dominant for $1040 \mathrm{~nm}$ pulse propagation in the CHG waveguide, while the linear dispersion is negligible.

The NLDC is then tested with both $p$-polarized and $s$-polarized inputs, and with laser pulses launched into port 1 and port 2, yielding four sets of experiment results. For each input polarization at low peak intensity $\left(5 \mathrm{GW} / \mathrm{cm}^{2}\right)$, the insertion loss for the NLDC is the same as that of the waveguide; the insertion loss rises to $\sim 5.9 \mathrm{~dB}$ when the input peak intensity is increased to $90 \mathrm{GW} / \mathrm{cm}^{2}$, due to nonlinear absorption of the material. The NLDC shows the characteristic curve of nonlinear optical switching, while the switching peak intensities and splitting ratios vary among the four conditions (Fig. 4).

Starting from low input peak intensity, due to linear evanescent mode coupling, the majority of input power is guided through the cross channel at this designed coupling length, until the input peak intensity reaches the switching point, where self-focusing from the Kerr nonlinearity of the material compensates the linear diffraction, and more input power is transmitted through the bar channel with the further increase of input intensity. Among the four experimental conditions, the minimum switching intensity is $16 \mathrm{GW} / \mathrm{cm}^{2}$ for $s$-polarized laser pulses launched to port 1 [Fig. 4(b)]. With a nonlinear refractive index up to 400 times that of silica [2], the NLDC in ChG has a switching peak intensity reduced
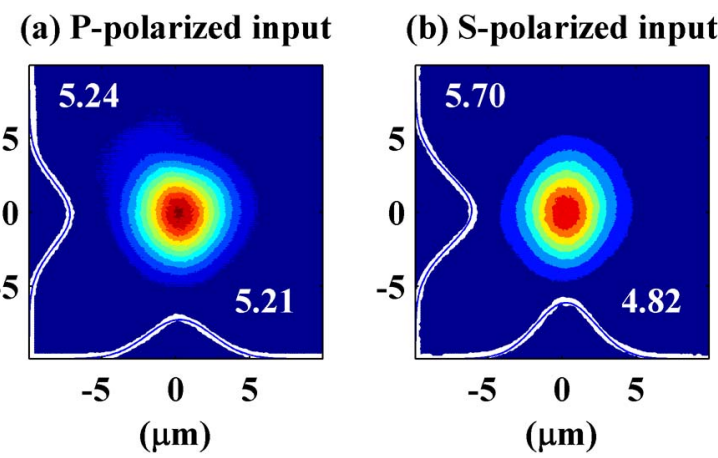

Fig. 3. Mode profiles of an ultrafast laser written waveguide in GLS glass with (a) $p$-polarized input and (b) $s$-polarized input. 
(a)

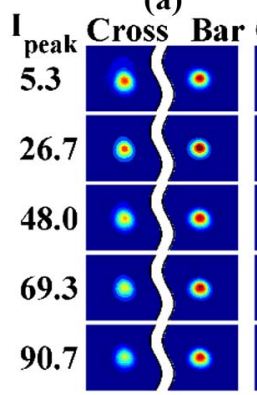

(b)

(c)

(d)
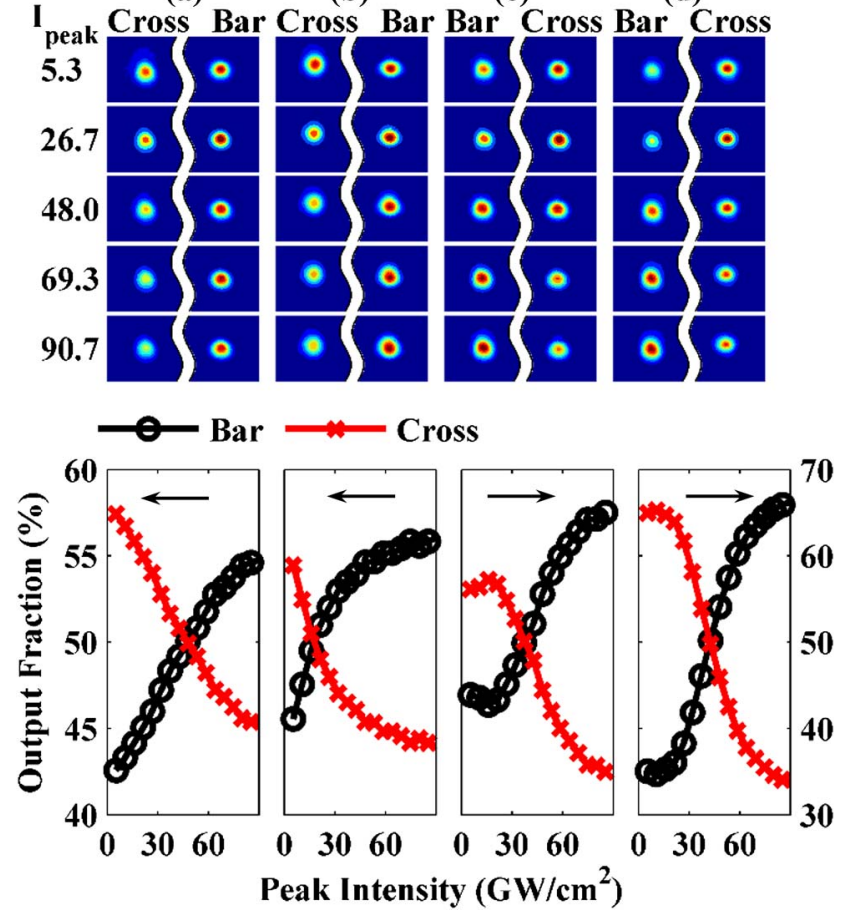

Fig. 4. Output mode profiles and power switching curves for different experiment conditions: input to port 1 (a) $p$-polarized, (b) $s$-polarized; input to port 2 (c) $p$-polarized, (d) $s$-polarized.

by two orders of magnitude, comparing to the NLDC demonstrated in silica $[12,13]$. The variation of the switching peak intensities and the split ratios among the four experimental conditions can be attributed to the birefringence and the asymmetry between the two waveguides, where the latter plays a major role as inferred from the resemblance between Figs. $\underline{4(\mathrm{a})}$ and $\underline{4(\mathrm{~b})}$, as well as between Figs. 4(c) and 4(d).

The output spectra and pulse widths of the two channels of the NLDC are also measured. Figure $\underline{5}$ shows the autocorrelation traces and the output spectra from both output ports of the NLDC when $p$-polarized pulses are coupled to port 2, corresponding to Fig. 4(c). The autocorrelation traces are measured when the input peak intensity is $90 \mathrm{GW} / \mathrm{cm}^{2}$, where the switching has occurred between the two ports and the power distribution is stabilized. In Fig. 5(a), compared to the input pulses, output pulses from both channels are stretched; the side wings are suppressed for the output pulse from the bar channel, since the center of pulse with higher intensity is confined within it while the sides with lower intensity are coupled to the cross channel; the output of the cross channel emerges as a doublet from the head and tail of the input pulse. From Figs. 5(b) and 5(c), propagation light from both channels experience spectral broadening. The spectral width is larger for output from the bar channel, as a larger fraction of power is transmitted through it at high input intensity. Besides, the spectral broadening is asymmetric for the output pulse from the cross channel, with more frequency components generated in the long wavelength side. This is because the switching power is related to wavelength by $P_{c}=\left(A \lambda / n_{2} L_{c}\right)$ [13], where
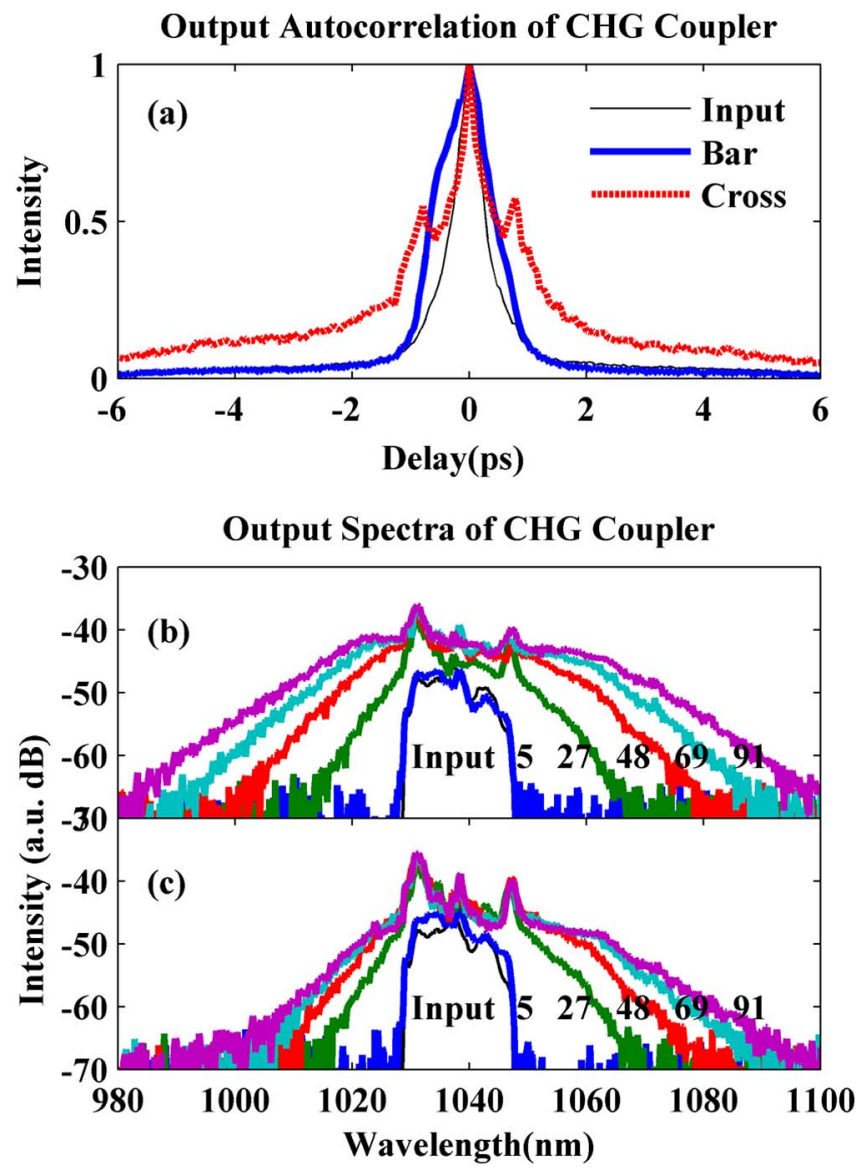

Fig. 5. (a) Autocorrelation traces of the input pulse and output pulses from the two coupler channels with the peak intensity of the input being $90 \mathrm{GW} / \mathrm{cm}^{2}$. [(b), (c)] Spectral evolution of the two coupler outputs, (b) Bar; (c) Cross. The input is $p$-polarized and coupled to port 2 of the NLDC. Each trace in the spectra is marked with the corresponding input peak intensity (unit: $\mathrm{GW} / \mathrm{cm}^{2}$ ).

$A$ is the effective mode area, $\lambda$ is the wavelength, $n_{2}$ is the nonlinear refractive index, and $L_{c}$ is the coupling length. Longer wavelength components require higher switching power; thus they tend to be coupled to the cross channel while the short wavelength components are switched to the bar channel, resulting in the asymmetry of the output spectrum.

In conclusion, a NLDC is fabricated in GLS chalcogenide glass by ultrafast laser. Compared to NLDC in silica, the ChG NLDC reported here has a reduced switching intensity of $16 \mathrm{GW} / \mathrm{cm}^{2}$. With increased input intensity, spectral width of the outputs of the NLDC is increased from $20 \mathrm{~nm}$ to up to $140 \mathrm{~nm}$, while pulse stretching is insignificant for the short propagation length. The ultrafast writing parameters can be further optimized to enhance the symmetry of the NLDC so as to obtain uniform performance regardless of the input polarization or the input port. This ChG NLDC demonstrates the potential of ultrafast laser in writing integrated photonics circuits in highly nonlinear material for improved performance and sets the groundwork to fabricate 3D optical components by ultrafast laser writing.

This work was supported by the National Science Foundation (CMMI-0900564 and CHE-0911456). 


\section{References}

1. B. J. Eggleton, B. Luther-Davies, and K. Richardson, Nat. Photonics 5, 141 (2011).

2. A. Zakery and S. R. Elliott, J. Non-Cryst. Solids 330, 1 (2003).

3. S. J. Madden, D. Y. Choi, D. A. Bulla, A. V. Rode, B. Luther-Davies, V. G. Ta'eed, M. D. Pelusi, and B. J. Eggleton, Opt. Express 15, 14414 (2007).

4. R. R. Gattass and E. Mazur, Nat. Photonics 2, 219 (2008).

5. A. M. Streltsov and N. F. Borrelli, Opt. Lett. 26, 42 (2001).

6. J. C. Ng, C. B. Li, P. R. Herman, and L. Qian, Opt. Express 20, 17894 (2012).

7. R. Keil, M. Heinrich, F. Dreisow, T. Pertsch, A. Tunnermann, S. Nolte, D. N. Christodoulides, and A. Szameit, Sci. Rep. 1, 6 (2011).
8. A. Szameit, F. Dreisow, T. Pertsch, S. Nolte, and A. Tünnermann, Opt. Express 15, 1579 (2007).

9. B. McMillen, B. T. Zhang, K. P. Chen, A. Benayas, and D. Jaque, Opt. Lett. 37, 1418 (2012).

10. N. D. Psaila, R. R. Thomson, H. T. Bookey, S. X. Shen, N. Chiodo, R. Osellame, G. Cerullo, A. Jha, and A. K. Kar, Opt. Express 15, 15776 (2007).

11. J. E. McCarthy, H. T. Bookey, N. D. Psaila, R. R. Thomson, and A. K. Kar, Opt. Express 20, 1545 (2012).

12. A. Betlej, S. Suntsov, K. G. Makris, L. Jankovic, D. N. Christodoulides, G. I. Stegeman, J. Fini, R. T. Bise, and J. DiGiovanni, Opt. Lett. 31, 1480 (2006).

13. S. R. Friberg, A. M. Weiner, Y. Silberberg, B. G. Sfez, and P. S. Smith, Opt. Lett. 13, 904 (1988). 